\title{
Polarization and Radiation Pattern Reconfigurability of a Planar Monopole-Fed Loop Antenna for GPS Application
}

\author{
Mohammad M. FAKHARIAN, Pejman REZAEI, Ali A. OROUJI \\ Dept. of Electrical and Computer Engineering, Semnan University, Semnan, 35131-19111, Iran \\ m_fakharian@semnan.ac.ir; prezaei@semnan.ac.ir; aliaorouji@semnan.ac.ir
}

Manuscript received February 29, 2016

\begin{abstract}
This paper presents a reconfigurable loop antenna with monopole-fed using embedded RF PIN based switches and shorted parasitic elements for GPS applications. The antenna can independently reconfigure multiple polarizations with switchable radiation pattern. Four switched metallic patches are used as parasitic elements to provide a reconfiguration capability to antenna acting as a driven monopole-fed loop. The edge of the parasitic elements is shorted by posts. The parasitic patches are connected/disconnected by using switching, therewith changing the configuration of monopole, to turn changes in the current distribution over the loop surface. The antenna is designed to work on the GPS $L_{1}$ frequency band. The antenna simultaneously changes the radiation beam in Eand H-planes, and switches among three polarizations (LP, LHCP, and RHCP) in the various modes. The antenna maximum gain among the different modes is tuned between 1.5 and $4.2 \mathrm{dBi}$.
\end{abstract}

\section{Keywords}

Loop antenna, reconfigurable, RF PIN switch, pattern diversity, polarization agile, monopole-fed

\section{Introduction}

Reconfigurable radio systems are the most promising ideas for wireless networks, which are the fundamental principle in cooperative networks and cognitive radios [1-4]. Antennas with radiation pattern reconfiguration can be used to avoid in-band interference, noisy environments, and increase the security and capacity of the system [5]. Polarization reconfiguration is also used to filter the detrimental fading loss caused by the multipath effect [6]. These reconfigurations are obtained by many techniques that redistribute the current flow on the antenna and thereby adapt to change the performance characteristics and electromagnetic (EM) fields of the antenna's effective aperture. Typically, these reconfiguration techniques are based on the integration of electronic switching compo- nents such as PIN diodes [5] on the radiating structure of the antenna.

Designs of the reconfigurable antenna with simultaneous function as a multimode antenna for polarization selectivity and radiation pattern diversity have been investigated so far [6-8]. In [6], a reconfigurable microstrip antenna is obtained by using a four-way power divider to feed different radiating elements. A microstrip-fed truncated monopole is arranged side by side with a common ground plane and is activated alternately to achieve the reconfigurable radiation pattern and polarization diversity in [7]. A reconfigurable antenna with sandwich topology with two switched crossed slots in ground plane has been proposed for MIMO system [8]. However, these works lead to a low reconfigurability and providing few operating modes with comparatively similar characteristics. On the other hand, these works are extremely complex in configuration and fabrication inconvenience with the usage of more vias and switching devices, which severely impacts the antenna cost and efficiency. Therefore, the design of a reconfigurable antenna, which can simultaneously incorporate different radiation pattern and polarization characteristics into a single antenna element, has become an important research area for antenna designers.

A Global Positioning System (GPS) system is required to operate with the single radiating element. The GPS device is limited by having a clear access to the satellite that provides the tracking information. The device signal cannot pass through dense material such as stone, concrete, metal, dirt, or thick trees. On certain locations, obstructed by tall buildings and with sparse coverage, the signal reception of up to $90 \%$ can be poor or even lost [9]. Furthermore, the position of the GPS antenna mounting is crucial for performance of the GPS receiver. The antenna must have full view of the sky ensuring a direct line-ofsight with as many visible satellites as possible. The antenna should be placed as far away as possible from radiating or jamming signals. As a solution, the reconfigurable antenna could be used to overcome the overall signal lost and their placement location, since the antennas become able to adapt to the changing system requirements and operating environment. 
For optimal performance of the GPS systems, we usually use antennas with high gain, low level of directivity, and right-hand circular polarized (RHCP) [10]. The signal transmitted from the satellites is RHCP and, therefore, the terminal antenna must also use RHCP in order to have the maximum received signal strength. However, reception occurs after the RHCP signal is reflected, or bounces off of a surface once or more times. The number of reflections must be an odd number. The left-hand circular polarized (LHCP) antenna may be mounted underneath a vehicle or a building overhang. Therefore a LHCP antenna for receiving a non-line of sight satellite GPS location signal and a surface, wherein the non-line of sight satellite GPS location signal is reflected from the surface, is required. Furthermore, a linearly polarized (LP) antenna can receive half of the $\mathrm{CP}$ wave energy (regardless of whether the wave is RHCP or LHCP), which equates to a power loss of $3 \mathrm{~dB}$. This type of the antenna can be used for system-on-package integration [11].

In this paper, a novel reconfigurable loop antenna with monopole-fed is designed. The reconfigurability of the antenna is achieved using four shorted parasitic elements which are connected to one driven element by four PIN diodes. The proposed antenna is capable of reconfiguring the antenna parameters: polarization, gain, and radiation pattern. This technique has been implemented to a planar monopole-loop structure, leading to small dimensions and low complexity. The size in total dimensions is less than $0.2 \lambda_{0}$ in the operation frequency. The antenna is designed to operate in the GPS $\mathrm{L}_{1}$ frequency band $(1.57 \mathrm{GHz})$ in six different states. A fully functional prototype is presented whose compact and accretion characteristics make it proper for GPS application. The design avoids a complicated biasing circuit and a tunable matching network.

\section{Antenna Structure and Working Mechanism}

Figure 1 shows the configuration of the proposed monopole-loop antenna with switched parasitic elements. It is printed on an FR4 dielectric substrate with relative permittivity 4.4 , thickness $1.6 \mathrm{~mm}$, tangential loss 0.02 , and size of $33 \times 30 \mathrm{~mm}^{2}\left(0.17 \lambda_{0} \times 0.16 \lambda_{0}, \lambda_{0}\right.$ being the freespace wavelength at the center frequency $1.57 \mathrm{GHz}$ ). The antenna includes a semi-rectangular loop printed on the substrate lower layer. The upper layer consists of a circular radiating monopole as a driven element in the center of the substrate and four parasitic elements marked as parasitic $\mathrm{P}_{1}, \mathrm{P}_{2}, \mathrm{P}_{3}$ and $\mathrm{P}_{4}$. All parasitic elements are located around the driven element in a circle surrounding. In order to increase the capacitive load, the distance between the parasitic elements and the driven monopole element is reduced, and thus the overall radius of the antenna is reduced. The parasitic elements are connected to the driven element through RF PIN diodes $\left(D_{1}, D_{2}, D_{3}\right.$ and $\left.D_{4}\right)$. It is noted that metallic vias are used to connect the parasitic elements to

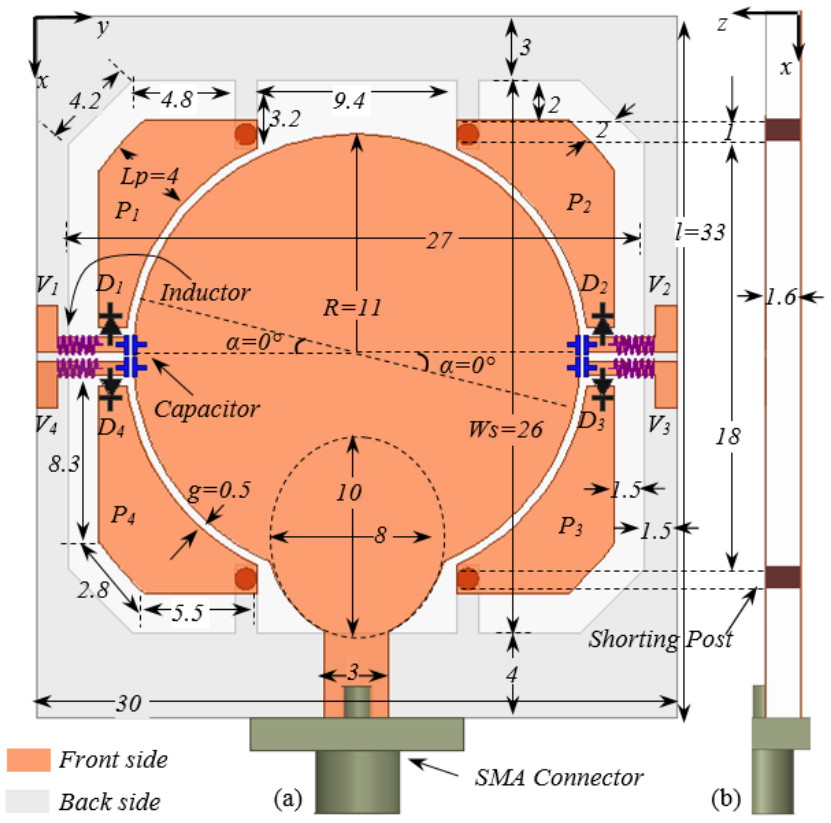

Fig. 1. Geometry of the proposed reconfigurable slot antenna structure: (a) top view; (b) side view (unit: $\mathrm{mm}$ ).

the back side. All of these parasitic elements are physically the same electrical length, switching their states between short and open circuited to the back plane leads to a variation of the elements' current distribution. The driven element is excited with a monopole feed line. The feed line is composed of a semi elliptical-shaped impedance transformer to give an optimum impedance matching.

To control the diode states, four independent DC bias voltages, $V_{1}, V_{2}, V_{3}$ and $V_{4}$ are applied to four terminals. In order to choke the RF signal in the feed line from entering the DC bias network, surface-mount inductors $(56 \mathrm{nH})$ are inserted in series with each DC feed line at each terminal. Surface-mount capacitors $(100 \mathrm{pF})$ are used to maintain the RF continuity and prevent DC short circuit of the PIN diodes.

The operation and design of the antenna is as follows. The starting point in the design of the antenna, in this work, is an ordinary rectangular loop antenna with a square shaped metallic surface acting as a monopole feed, as shown in Fig. 2(a). Based on the concept of the pixel antenna [12], the parsing the stub surface into nonuniform electrically small parts are considered. These parts are interconnected with RF PIN diodes as switching devices, thus a circular shape metallic main radiator surrounded by four identical parasitic elements is achieved, as shown in Fig. 2(b). This location and dimensions of the parasitic elements are designed to maximize the coupling with the driven element and lead to a compact structure. In the following, we use a shorting post on the other side of the parasitic elements, as shown in Fig. 2(c), which elements become a section of a loop antenna when connected to the loop on the back side, so they act as a longer loop with larger electrical length. When these parasitic elements with shorting posts are connected to the driven monopole using diodes, the antenna operates in the various loop modes. 


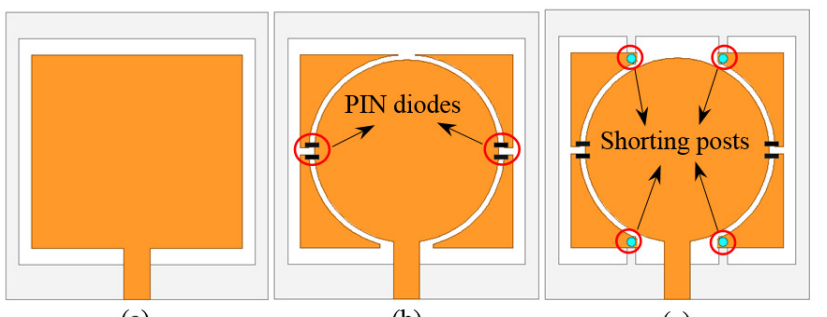

(a)

(b)

(c)

Fig. 2. Progression of perceptual steps followed during the substantiation of the monopole-loop antenna with switched parasitic elements: (a) ordinary loop antenna, (b) decomposed monopole surface into four metallic pieces and reconfigurable surface by interconnecting the pieces with four switches, and (c) inserting four shorting posts into the parasitic elements.

When all the parasitic elements are disconnected from the driven monopole, the antenna operates in the loop mode with the difference in the radiation characteristics. On the other hand, the shorted parasitic elements are switched to each edge of the driven monopole. So they can be acted as a shorting wall that represses the E-field at the center of that edge. As a result, the maximum level of power in one plane is lower than that in other plane [6]. Four shorting strips are used at the top and bottom of the slot, which play a minor role in reducing the frequency gap of the modes.

Finally, to modify the antenna performance for the loop mode operation and circular polarization states, a wide parametric study is carried out, and the final dimensions of the antenna are specified, as shown in Fig. 1. A semi elliptical-shaped impedance transformer has been added to the feeding line. This type of the impedance transformer plays a significant role in the tuning the effects of EM coupling between the loop and the monopole feed, and it furthers its impedance matching without any cost of expense or size. Furthermore, a desired matching level in the driven monopole is obtained by adapting its relative position with respect to the parasitic elements.

Therefore, the antenna can be operated as a multicharacterized antenna to have different radiation patterns and polarizations while retaining the same operating frequency. The working principle of the proposed antenna is been shown and listed in Tab. 1 with respect to beam directions (BDs) and polarizations by proper selecting the switch configuration.

\section{Simulated and Measured Results and Discussions}

In this section, a prototype of the antenna was fabricated and measured, and the experimental and numerical results of the input reflection coefficient $\left(\left|\mathrm{S}_{11}\right|\right)$ and radiation characteristics are presented and discussed. For the proposed antenna, the low-cost PIN diodes (Infineon Techn. BAR50-02V) are used. Metallic pads are etched on the substrate to allow mounting of the inductors, capacitors and PIN diodes. Based on the applied PIN diode data-

\begin{tabular}{|c|c|c|c|c|c|}
\hline \multirow{2}{*}{ State } & \multicolumn{4}{|c|}{$\begin{array}{c}\text { PIN Diode } \\
\text { Operation }\end{array}$} & Operation mode at the GPS band \\
\hline & D1 & D2 & D3 & D4 & \\
\hline A & 1 & 0 & 0 & 0 & LP, BD at $\phi=75^{\circ}(\mathrm{x}-\mathrm{y}), \theta=15^{\circ}(\mathrm{x}-\mathrm{z})$ \\
\hline $\mathrm{A}^{\prime}$ & 0 & 1 & 0 & 0 & $\mathrm{LP}, \mathrm{BD}$ at $\phi=285^{\circ}, \theta=-15^{\circ}(\mathrm{x}-\mathrm{z})$ \\
\hline B & 1 & 0 & 0 & 1 & LP, BD at $\phi=50^{\circ}, \theta=25^{\circ}(\mathrm{x}-\mathrm{z})$ \\
\hline B $^{\prime}$ & 0 & 1 & 1 & 0 & LP, BD at $\phi=310^{\circ}, \theta=-25^{\circ}(\mathrm{x}-\mathrm{z})$ \\
\hline C & 1 & 0 & 1 & 0 & RHCP, BD at $\phi=95^{\circ}, \theta=-30^{\circ}(\mathrm{x}-\mathrm{z})$ \\
\hline $\mathrm{C}^{\prime}$ & 0 & 1 & 0 & 1 & LHCP, BD at $\phi=265^{\circ}, \theta=30^{\circ}(\mathrm{x}-\mathrm{z})$ \\
\hline
\end{tabular}

Tab. 1. Antenna possible configurations (0: OFF and 1: ON).

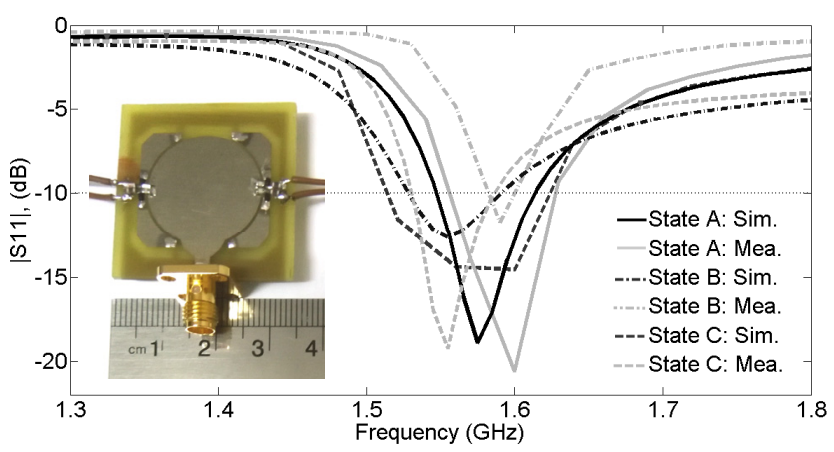

Fig. 3. Simulated and measured $|\mathrm{S} 11|$ of the different antenna states, as listed in Tab. 1.

\begin{tabular}{|c|c|c|}
\hline State & Simulated & Measured \\
\hline $\mathrm{A}$ & $1.550-1.610 \mathrm{GHz}, 3.8 \%$ & $1.555-1.625 \mathrm{GHz}, 4.4 \%$ \\
\hline $\mathrm{B}$ & $1.530-1.592 \mathrm{GHz}, 4.0 \%$ & $1.570-1.60 \mathrm{GHz}, 1.9 \%$ \\
\hline $\mathrm{C}$ & $1.515-1.625 \mathrm{GHz}, 7.3 \%$ & $1.53-1.585 \mathrm{GHz}, 3.5 \%$ \\
\hline
\end{tabular}

Tab. 2. Antenna impedance bandwidth simulated vs. measured results.

sheet [13], it has a forward resistance of $R_{\mathrm{S}}=3 \Omega$ for the ON state and a parallel circuit with a capacitance of $C_{\mathrm{T}}=0.15 \mathrm{pF}$ and a resistance of about $R_{\mathrm{P}}=5 \mathrm{k} \Omega$ for the OFF state. These effects can be modeled by using the RF equivalent circuit in the full-wave simulations in the ON/OFF states using Ansoft's HFSS.

The input reflection coefficient $\left(\left|S_{11}\right|\right)$ of the proposed antenna, according to Tab. 1, is shown in Fig. 3 for the simulated and measured results, respectively. However, due to the antenna symmetry structure, the $\left|S_{11}\right|$ results for $\mathrm{A}^{\prime}, \mathrm{B}^{\prime}$, and $\mathrm{C}^{\prime}$ are not given here as they have the same results of $\mathrm{A}, \mathrm{B}$, and $\mathrm{C}$, respectively. The simulated and measured results are slightly different owing to fabrication tolerance, material loss, SMA connector, via process and the actual DC biasing circuitry. Although the frequencies shift slightly with respect to the frequencies observed in the simulation results, the requirement of the $10-\mathrm{dB}$ bandwidth is satisfied by the variation from $1.55 \mathrm{GHz}$ to $1.6 \mathrm{GHz}$. It is verified that the proposed antenna satisfactorily operates at the GPS bandwidth $(1575 \mathrm{MHz} \pm 1 \mathrm{MHz})$. The achieved impedance bandwidth $\left(\left|S_{11}\right| \leq-10 \mathrm{~dB}\right)$ achieved from simulation data in comparison to measurements is tabulated in Tab. 2.

A loop antenna generally operates at one-wavelength mode. After modifying the slot width, the length of 

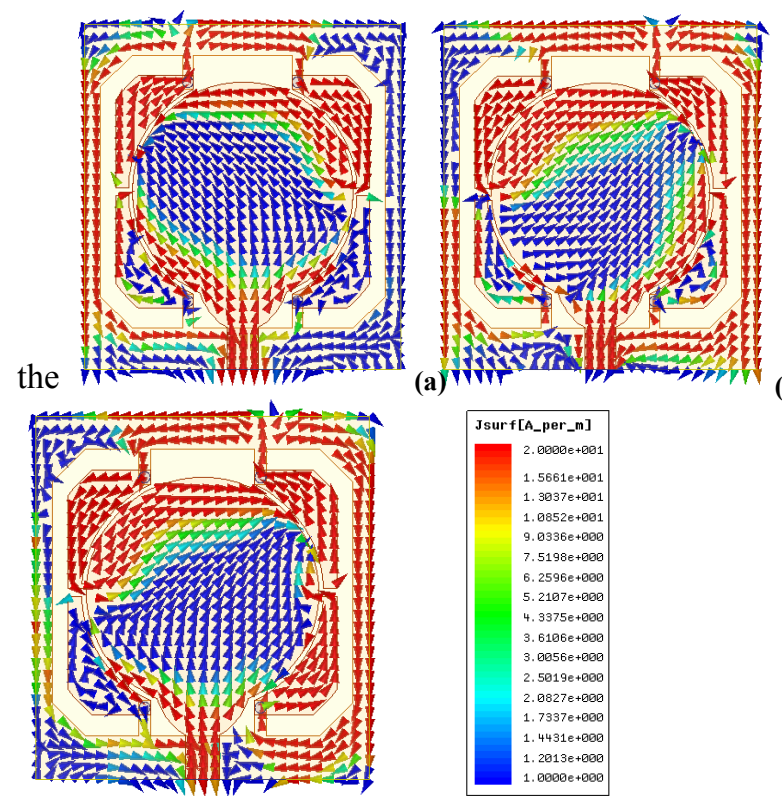

(c)

Fig. 4. Current distributions of the antenna in the various states at 1.57 GHz: (a) state-A; (b) state-B; (c) state-C.

semi-rectangular loop on the bottom plane could be tuned to make sure that the loop works in the desired mode [14]. Figure 4 shows the antenna current distributions in the various states at $1.57 \mathrm{GHz}$. As shown, the current distribution is mostly concentrated at the loop edges. The current is flowed from the monopole and parasitic elements to the loop, and the distributions and intensity of current are different at the various states of antenna, while they are of the same electrical length.

According to the above discussions, polarization reconfigurability is possible in the design by creating strong transverse currents. Although currents over the driven and parasitic patches are mainly y-oriented, strong x-oriented currents can be induced by creating cross-shaped sections over the surface using the diodes. In this way, adjusting to the magnitude balance and phase shift between $\mathrm{x}$ and $\mathrm{y}$ currents, it is possible to provide also LP, RHCP and LHCP. For example, at B- and B'-states, the two LP can be switched electrically, and each is perpendicular to the other. These antennas have bilaterally symmetrical structures, and thus exhibit orthogonal polarization at the same working frequency. At $\mathrm{C}$-state, the shape of the antenna is modified with two short circuits from driven monopole stub to parasitic elements $\mathrm{P}_{1}$ and $\mathrm{P}_{3}$ using PIN diodes $\mathrm{D}_{1}$ and $\mathrm{D}_{3}$, which makes the structure asymmetric with respect to the horizontal and vertical central lines. In this case, the two short circuits force the currents to flow through the center of the driven stub to parasitic elements as well as along the edges of them. This behavior results in the splitting of the current into two near orthogonal resonant modes along the $\mathrm{y}$ - and $\mathrm{x}$-direction, respectively.

Because the amplitude ratio and phase difference of the two orthogonal resonant modes depend on the field distributions along the short circuits and driven patch, it is reasonable to assume that the $\mathrm{CP}$ operation is related to

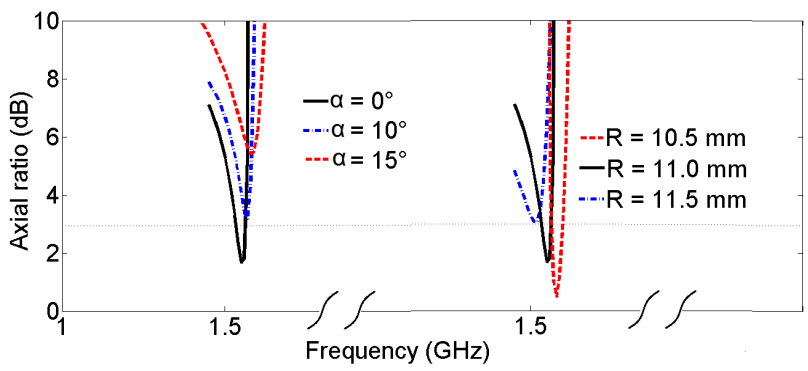

Fig. 5. AR of the C-state for the effects of various $\alpha$ and $R$.

position of diodes and capacitors $(\alpha)$ and radius of the driven patch $(R)$. Figure 5 shows the effects of $\alpha$ and $R$ on the AR of the C-state. As seen from Fig. 5, the frequency of minimum ARs with the center frequencies of 1.53 and $5.1 \mathrm{GHz}$ shift to higher frequency as $\alpha$ increases, while the 3-dB AR bandwidth decreases. This corroborates that the antenna surface is able to produce strong currents in the transverse direction. It seems that the horizontal position of PIN diodes has effect on both current amplitude and phase shift that are sensitive to bandwidth and frequency of $\mathrm{CP}$ mode, respectively. Therefore, $\alpha$ has the function of fine tuning to broaden CP bandwidth and center frequency. As seen from this figure, the 3-dB AR bandwidth increases for higher bands and decreases for lower bands as $R$ increases. However, the operation frequency of the lowest AR is inverse with $R$ for lower bands and is consilient for the higher bands. Therefore, in this design, the effect of amplitudes of two orthogonal fields limits $\mathrm{CP}$ operation and degrades the AR bandwidth. It can be also seen that the $\mathrm{CP}$ operating frequency bands are all located within the range from 1.5 to $1.6 \mathrm{GHz}$ as $\alpha$ and $R$ are varied, which is wide enough to cover the GPS $1.57 \mathrm{GHz}$ frequency band. Correspondingly, the same judgments apply in the LHCP case for the $\mathrm{C}^{\prime}$-state.

Figure 6 presents the simulated and measured crosspolarization levels (XPL) for the linear polarization (LP) modes and the axial ratios (ARs) for the circular polarization (CP) modes at the broadside direction. Satisfactory agreements are achieved between the simulated and measured results. The difference between them may come from the efficacy of the fabrication error. The left hand (LH) and right hand circular polarization (RHCP) modes have the

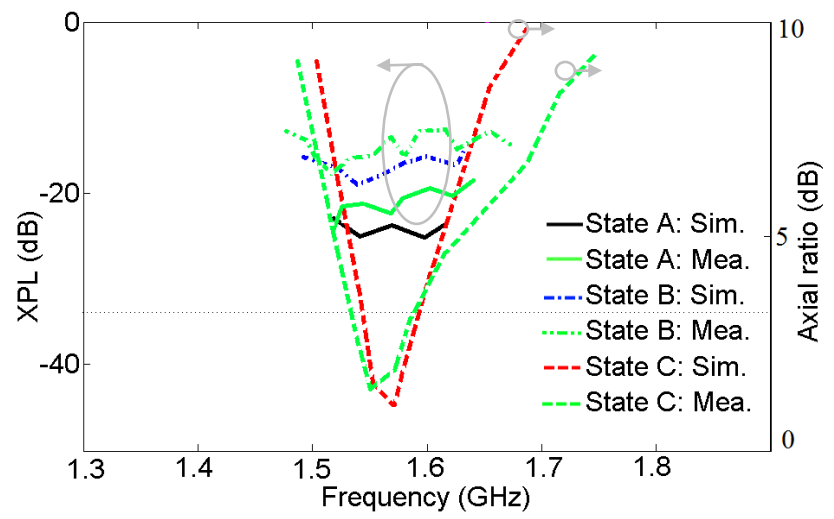

Fig. 6. Simulated and measured XPL and AR of the different antenna states, as listed in Tab. 1. 
similar results in the same resonance frequency, because of structure symmetry. Thus, only the RHCP mode results are given for brevity. Furthermore, the results for $\mathrm{A}^{\prime}, \mathrm{B}^{\prime}$, and $\mathrm{C}^{\prime}$ are not given here as they have the same results of $\mathrm{A}, \mathrm{B}$, and $\mathrm{C}$, respectively. For all LP modes, the XPLs within their respective impedance bandwidths are less than $16 \mathrm{~dB}$, which shows a good isolation between LP and CP modes. The achieved CP bandwidths, referred to 3-dB AR, calculated from simulation in comparison to measurements for the C-state are $1.54-1.595 \mathrm{GHz}$ and $1.535-1.592 \mathrm{GHz}$, respectively. It has to be noted that the impedance bandwidths in Tab. 2 totally cover the corresponding AR bandwidth in this state.

Figure 7 shows only the measured and simulated radiation patterns normalized to their own maxima at $1.57 \mathrm{GHz}$ for $\mathrm{C}$ - and $\mathrm{C}^{\prime}$-states, where good agreement between the simulated and measured results exists. It is also observed that the radiation pattern is slightly tilted offbroadside by about $8^{\circ}$. The beam tilt is due to the asymmetrical geometry of the patch antenna, compared to a plain patch which has a symmetrical geometry and exhibits a broadside beam. The major polarization $\mathrm{Z}>0$ is the LHCP and it is the RHCP for $\mathrm{Z}<0$. The CP sense can be determined by the left-hand rule, in which the fingers rotate in accordance with the flow path of the current on the antenna.

The antenna operates in the loop mode for all states by switching between the diode different states. In these designs, all parasitic elements are positioned in the direction of 45, 135, 225 and 315 degrees to the driven radiator. The parasitic elements are connected via a shorting post to
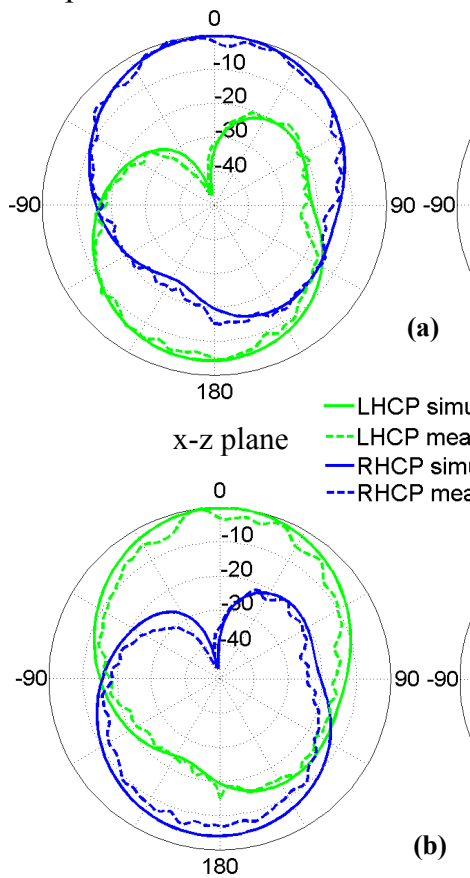

(b)
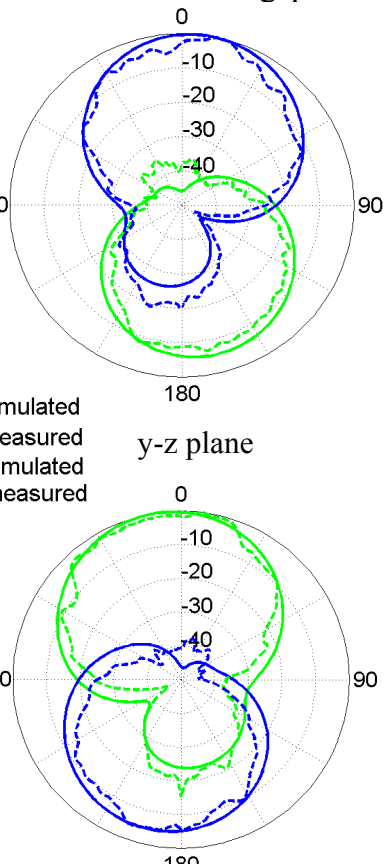

180
Fig. 7. Measured and simulated E-plane ( $x-z$ plane) and $\mathrm{H}$-plane (y-z plane) normalized radiation patterns at $1.57 \mathrm{GHz}$ for a) $\mathrm{C}$ - and b) $\mathrm{C}^{\prime}$-states.

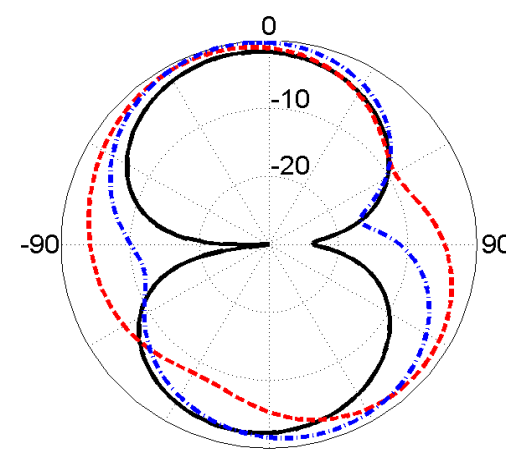

180

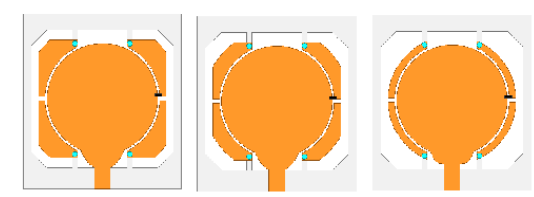

$-L p=4 \mathrm{~mm}=-\mathrm{Lp}=2.5 \mathrm{~mm}=-\mathrm{Lp}=1.2 \mathrm{~mm}$

Fig. 8. Antenna radiation pattern in the $y-z$-plane at $A^{\prime}$-state for three different lengths of the parasitic patch (Lp).

the loop on the back plane. Due to the EM energy created by the driven monopole antenna, the impedance loading occurs and it couples to the parasitic elements with the mutual and direct coupling of EM energy. The surface current on them can resonate concluding in radiation at a particular direction, when the parasitic elements are loaded with suitable reactive loads. Thus, the induced currents on them are maximized when their sizes near the resonant size. In the design, the equivalently reactive loads or mutual impedances can be different by changing the relative locations and shapes of the parasitic elements, which eventually results in the different modes of operation corresponding to beam steering angles and various polarizations at the same frequency range. Figure 8 shows the radiation patterns for $\mathrm{A}^{\prime}$-state with different parasitic shapes. The antenna has a bandwidth from 1.52 to $1.6 \mathrm{GHz}$ for all parasitic shapes at lower mode. At $1.57 \mathrm{GHz}$, the $\mathrm{H}$-plane $\mathrm{BD}$ is steered smoothly from $0^{\circ}$ to $27^{\circ}$ when the radial sector parasitic decreases from 4 to $1.2 \mathrm{~mm}$ in three cases, while the E-plane pattern is broadside.

The simulated 3-D radiation patterns at the center of the frequency range for $\mathrm{x}-\mathrm{z}$ and $\mathrm{y}-\mathrm{z}$ planes are presented in Fig. 9. The antenna can change the beam in both $x-z$ and $y-z$ planes. The presented design can generate common loop-like radiation pattern. The maximum gain for the radiation pattern steered to $-\mathrm{x}$-axis and $+\mathrm{x}$-axis is $4.2 \mathrm{dBi}$, and it can be tuned between $1.5 \mathrm{dBi}$ for $\mathrm{A} / \mathrm{A}^{\prime}$ states, $4.2 \mathrm{dBi}$ for $\mathrm{B} / \mathrm{B}^{\prime}$ states, and $2.9 \mathrm{dBi}$ for $\mathrm{C} / \mathrm{C}^{\prime}$ states. The differences in gain are basically due to some configurations producing a polarization different from that of the patch antenna and higher losses due to polarization mismatch. The average radiation efficiency of these states is $55 \%$, which has been computed comparing the antenna gain and directivity and is due almost entirely to the PIN diode losses. A larger ground plane will increase the gain of the antenna, but it will also affect in the bandwidth frequencies. 


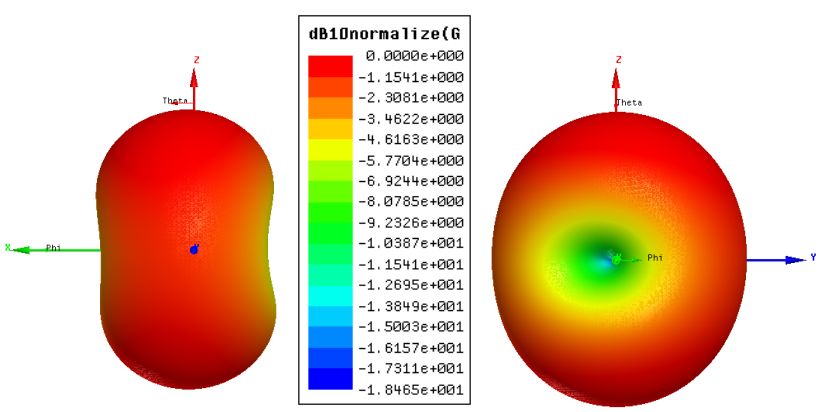

(a)
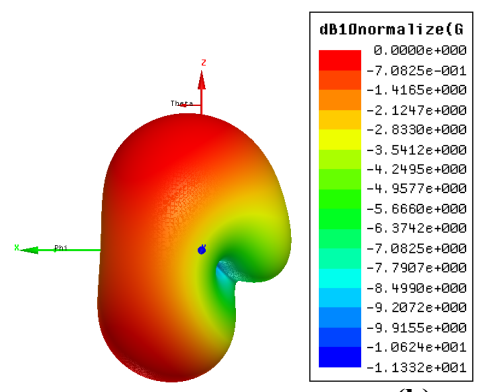

(b)

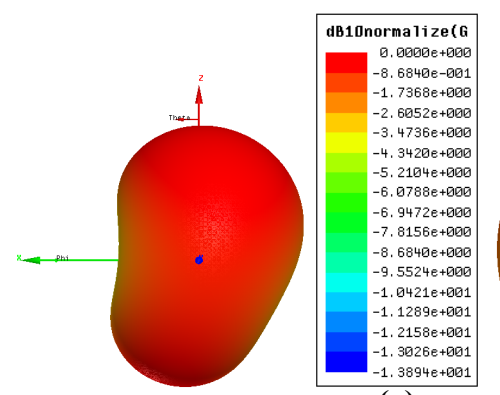

(c)
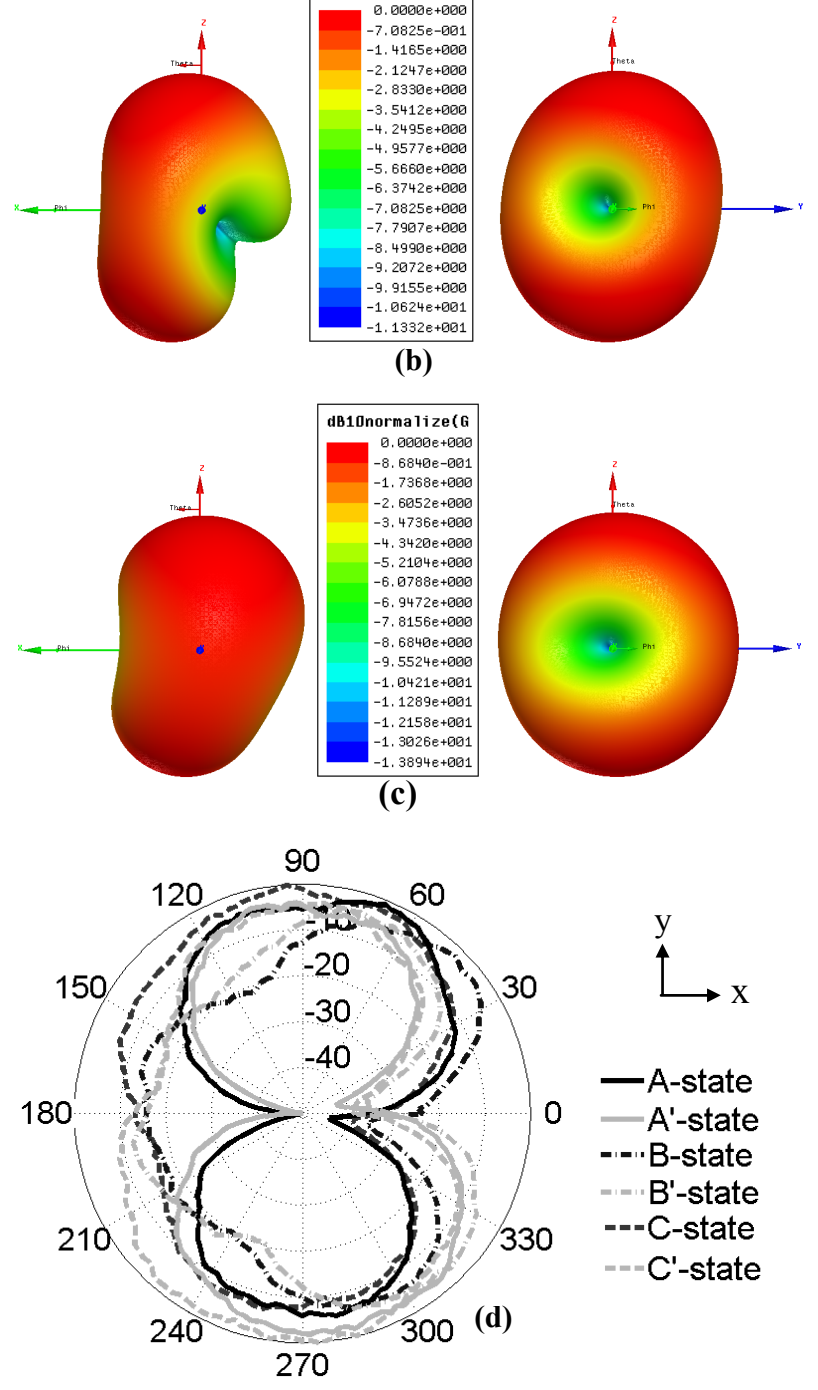

Fig. 9. Simulated 3-D radiation patterns in both $x-z$ and $y-z$ planes of (a) A-state, (b) B-state, and (c) C-state. (d) Measured 2-D pattern in $\mathrm{x}-\mathrm{y}$ plane at $1.57 \mathrm{GHz}$ for various states.

\section{Conclusion}

A reconfiguration capability has been achieved by a planar monopole-loop antenna with the shorted parasitic elements. It has been demonstrated that the proposed antenna can simultaneously function as a multimode antenna for polarization agile and radiation pattern diversity systems. A functional prototype using integrated four PIN diodes was fabricated to illustrate the principles of working, and also to show its feasibility for fabrication. The fabricated antenna can operate at around $1.57 \mathrm{GHz}$ band by configuring the combination of the switch states. The antenna can also radiate horizontal, vertical or RH/LHCP in this band. The radiation pattern shape is examined for the different states. The proposed antenna is a demonstration of a single reconfigurable monopole-loop antenna using PIN diodes for potential use in stationary terminals of GPS applications.

\section{Acknowledgments}

The authors would like to thank all the members of the Antenna Laboratory at Iran Telecommunication Research Center (ITRC), for their cooperation. Also, the authors would like to thank the anonymous reviewers for their constructive comments.

\section{References}

[1] FAKHARIAN, M. M., REZAEI, P., OROUJI, A. A., et al. A wideband and reconfigurable filtering slot antenna. IEEE Antennas and Wireless Propagation Letters. Early Access Articles, DOI: 10.1109/LAWP.2016.2518859

[2] FAKHARIAN, M. M., REZAEI, P., OROUJI, A. A Reconfigurable multiband extended U-slot antenna with switchable polarization for wireless applications. IEEE Antennas and Propagation Magazine, 2015, vol. 57, no. 2, p. 194-202. DOI: 0.1109/MAP.2015.2414665

[3] FAKHARIAN, M. M., REZAEI, P., OROUJI, A. A. A novel slot antenna with reconfigurable meander-slot DGS for cognitive radio applications. Applied Computational Electromagnetics Society Journal, 2015, vol. 30, no. 7, p. 748-753.

[4] BORHANI KAKHKI, M., REZAEI, P., SHARBATI, V., et al. Small square reconfigurable antenna with switchable single/triband functions. Radioengineering, 2016, vol. 25, no. 1, p. 40-45. DOI: $10.13164 /$ re.2016.004

[5] QIN, P.-Y., GUO, Y. J., WEILY, A. R., et al. A pattern reconfigurable U-slot antenna and its applications in MIMO systems. IEEE Transactions on Antennas and Propagation, 2012, vol. 60 , no. 2 , p. 516-528. DOI: 10.1109/TAP.2011.2173439

[6] CAO, W., ZHANG, B., LIU, A., et al. A reconfigurable microstrip antenna with radiation pattern selectivity and polarization diversity. IEEE Antennas and Wireless Propagation Letters, 2012, vol. 11, p. 454-456. DOI: 10.1109/LAWP.2012.2193549

[7] RAMAN, P., MOHANAN, P., TIMMONS, N., et al. Microstripfed pattern- and polarization-reconfigurable compact truncated monopole antenna. IEEE Antennas and Wireless Propagation Letters, 2013, vol. 12, p. 710-713. DOI: 10.1109/LAWP.2013.2263983

[8] CHEN, W., SUN, J., FENG, Z. A novel compact reconfigurable polarization and pattern antenna. Microwave and Optical Technology Letters, 2007, vol. 49, no. 11, p. 2802-2805. DOI: $10.1002 /$ mop. 22865 
[9] AGARWAL, K., NASIMUDDIN, AROKIASWAMI, A. Tripleband compact circularly polarised stacked microstrip antenna over reactive impedance meta-surface for GPS applications. IET Microwaves, Antennas and Propagation, 2014, vol. 8, no. 13, p. 1057-1065. DOI: 10.1049/iet-map.2013.0586

[10] SYMMETRICOM, INC. General GPS Antenna Information. Application Note. [Online] Cited 2016-02-29. Available at: http://www.microsemi.com/document-portal/doc_view/133239general-gps-antenna-information

[11] BRZEZINA, G., ROY, L. A miniaturized GPS antenna in LTCC with linear polarization suitable for SoP integration. In 14th International Symposium on Antenna Technology and Applied Electromagnetics and the American Electromagnetics Conference (ANTEM-AMEREM). Ottawa (ON), 2010, p. 1-4. DOI: 10.1109/ANTEM.2010.5552526

[12] RODRIGO, D., JOFRE, L. Frequency and radiation pattern reconfigurability of a multi-size pixel antenna. IEEE Transactions on Antennas and Propagation, 2012, vol. 60, no. 5, p. 2219-2225. DOI: 10.1109/TAP.2012.2189739

[13] INFINEON TECHNOLOGIES. BAR50 Silicon PIN Diode (datasheet). 16 pages. [Online] Cited 2011-07-18. Available at: http://www.alldatasheet.com/datasheetpdf/pdf/78976/INFINEON/ BAR5002.html

[14] PAN, G., LI, Y., ZHANG, Z., FENG, Z. A compact wideband slotloop hybrid antenna with a monopole feed. IEEE Transactions on Antennas and Propagation, 2014, vol. 62, no. 7, p. 3864-3868. DOI: 10.1109/TAP.2014.2320535

\section{About the Authors ...}

Mohammad M. FAKHARIAN was born in Tehran, Iran, in 1987. He received the B.S. and M.S. degrees in Electrical Engineering from Semnan University, Semnan, Iran, in
2009 and 2012, respectively. Currently, he is working towards the Ph.D. degree in Communication Engineering from Semnan University. His research interests include low-profile printed and patch antennas for wireless communication, fractal, miniature and multiband antennas, meta-materials and EBG structures interaction with antennas and RF passive components, reconfigurable antennas, and electromagnetic theory: numerical methods and optimization techniques.

Pejman REZAEI was born in Tehran, Iran, in 1977. He received the B.S. degree in Electrical-Communication Engineering from Communication Faculty, Tehran, Iran, in 2000, and the M.S. and Ph.D. degrees from Tarbiat Modarres University, Tehran, Iran, in 2002 and 2007, respectively. Currently, he is associated professor in the Semnan University, Semnan, Iran. His current research interests are electromagnetics theory, theory and design of antenna, reconfigurable antenna, metamaterial structure, and satellite communication.

Ali A. OROUJI was born in Neyshabour, Iran, in 1966. He received the B.S. and M.S. degrees in Electronic Engineering from Iran University of Science and Technology, Tehran, Iran, in 1989 and 1992, respectively, and the Ph.D. degree from the Indian Institute of Technology Delhi, Delhi, India, in 2006. Since 1992, he has been a Faculty Member with Semnan University, Semnan, Iran. His research interests are in modeling of silicon-on-insulator metal-oxide-semiconductor field-effect transistors, novel device structures, theory and design of antenna, and wave propagation. 